\title{
PRINCIPALES FITÓFAGOS DE LA COCONA Solanum sessiliforum Dunal (SOLANACEAE) EN LA AMAZONÍA PERUANA
}

\author{
César DELGADO ${ }^{1}$, Guy COUTURIER ${ }^{2}$, Miguel ANTEPARRA $^{1}$ \\ 1 Instituto de Investigaciones de la Amazonía Peruana (IIAP), Programa de Investigación en Biodiversidad Amazónica (PIBA). \\ Iquitos, Perú, cdelgado@iiap.org.pe \\ 2 Institut de Recherche pour le Développement (IRD).
}

\section{RESUMEN}

Fueron encontrados 14 insectos y 1 nemátodo fitófago asociado con la cocona Solanum sessiliforum Dunal en la Amazonía peruana en el curso de evaluaciones realizadas entre mayo del 2009 y junio del 2012. Neoleucinodes elegantalis, Epicauta pestifera, Chromacris peruviana, Corythaica cyathicollis y Melodogyne sp. son las especies de mayor importancia para el cultivo, por los niveles de daño y la frecuencia con que se presentan en las parcelas tradicionales. La información se complementa con algunos datos sobre la biología y ecología para cada uno de ellos.

PALABRAS CLAVE: Solanum sessiliforum, fitofagos, Amazonía peruana, parcelas.

\section{PRINCIPAL HERBIVORES OF THE COCONA Solanum sessiliforum Dunal (SOLANACEAE) IN THE PERUVIAN AMAZON}

\begin{abstract}
Fourteen herbivorous insects and one phytophagous nematode were found in association with the cocona Solanum sessiliforum Dunal. in the Peruvian Amazon between May 2009 and June 2012. Neoleucinodes elegantalis, Epicauta pestifera, Chromacris peruviana, Corythaica cyathicollis and Melodogyne sp. are the most important pest species based on the frequency and extent of damage in our plots. Our research is supported by existing data on the biology and ecology of each of these species.
\end{abstract}

KEYWORDS: Solanum sessiliforum, phytophagous, Peruvian Amazon, plots. 


\section{INTRODUCCIÓN}

Solanum sessiliforum Dunal (Solanaceae), conocido como cocona en Perú, cubiu en Brasil y topiro por los indios del Alto Orinoco, de donde es originaria (Shultes, 1984). La cocona es una planta arbustiva, crece espontáneamente en lugares con mucha luz, como chacras, purmas, cultivos abandonados, claros en el bosque y bordes del bosque primario. El fruto es utilizado tradicionalmente para la preparación de jugos, dulces y mermeladas (TCA, 1998). La fruta es considerada como un alimento altamente dietético debido su bajo aporte calórico ( $45 \mathrm{kcal} / 100$ gr pulpa) y contenido significativo de fibras alimenticias (1.6 $\mathrm{g} / 100 \mathrm{~g}$ pulpa); esta evidencia sugiere su indicación en las mas variadas formas de consumo alimenticio por el poblador Amazónico, en especial en los pacientes hipercolesterolémicos e hiperglicémicos (Yuyama et al., 1997). En la medicina tradicional, las hojas se usan para evitar la formación de ampollas provocados por quemaduras, y el jugo de los frutos para calmar la picazón de la piel (TCA, 1998); también es usado para controlar colesterol, la diabetes, el exceso de ácido úrico y otras enfermedades causadas por el mal funcionamiento de los riñones y del hígado (Salick, 1987). Los mestizos y los indígenas peruanos utilizan el jugo de la cocona para dar brillo a los cabellos (Silva Filho, 1996). Probablemente algunas vitaminas y la pectina sean responsables de esta situación.

Hoy en día, en la Amazonía peruana, la cocona hace parte la emergente y variada gastronomía en forma de jugos, helados y salsas, que se expenden en los principales restaurantes turísticos.

Existen estudios relacionados con la cocona en aspectos como agronomía, industrialización y química. En estos últimos años el Instituto de Investigaciones de la Amazonía Peruana está trabajando en el mejoramiento genético de la especie (Balcazar et al., 2011); sin embargo, su desarrollo se ve limitado por la presencia de numerosos insectos fitófagos que atacan a la planta, como fue reportado para la Amazonía brasilera (Couturier, 1988).

\section{MATERIAL Y MÉTODOS}

El trabajo fue realizado de marzo de 2009 a junio de 2012, en las parcelas tradicionales las comunidades de Chingana, Flor de Castaña y Jenaro Herrera, en el Bajo río Ucayali, Loreto; en parcelas de colonos en los km 45 y 75 de la carretera Iquitos - Nauta, Loreto; y en parcelas de colonos en las comunidades El Milagro y Tulumayo, en Tingo María - Huanuco. Los insectos fueron colectados directamente de los órganos afectados de las plantas. En algunos casos, para facilitar la identificación, fue necesario transportar al laboratorio las estadios inmaduros de los insectos, para proceder a criarlos hasta obtener los adultos. Los especimenes fueron conservados en su mayoría al seco, y en menor proporción en alcohol glicerinado al $75 \%$. La identificación de las muestras fue realizado mediante revisiones bibliografías, comparación en las colecciones de los Museos, así como algunas muestras fueron enviados a especialistas de diferentes grupos taxonómicos.

\section{RESULTADOS}

Durante el estudio fueron registrados 14 insectos y un nematodos que causan daño a diferentes órganos de la cocona. Cinco especies son consideradas de mayor importancia para el cultivo de la planta en la Amazonía peruana; cuatro de ellas son nuevos registros para el cultivo: Neoleucinodes elegantalis, Epicauta pestifera, Chromacris peruviana y Melodogyne sp. Estas especies son diferentes a las encontradas en la Amazonía brasileña (Couturier, 1988).

A continuación se describe cada una de estas especies.

\section{Epicauta pestifera (Coleoptera: Meloidae).}

Escarabajos de color negro, cilindriforme, con la cabeza dirigida hacia abajo, de 19 a $31 \mathrm{~mm}$ de largo (Figura 1A). Alas no esclerotizadas, bien desarrolladas, que sobrepasan notablemente la longitud del abdomen. El adulto vive en colonias, se alimenta de las hojas, y en algunos casos de las inflorescencias de la planta. Cuando la colonia es densa en unas horas provocan la defoliación total de la planta. El adulto de este grupo secreta, como mecanismo de defensa, una sustancia muy irritante conocida como "cantaridina", que provoca ampollas al contacto con la piel. El huevo es depositado en el suelo, donde se desarrolla la larva hasta transformarse en adulto. Se alimenta durante el día. Esta especie fue encontrada provocando grandes defoliación a los sembríos de cocona en las localidades del bajo Ucayali, tanto en zonas inundables como en tierra firme.

\section{Alcidion deletum Bates (Coleóptera: Cerambicidae).}

El adulto es un escarabajo de color crema a ligeramente oscuro, mide de 15 a $20 \mathrm{~mm}$ de largo. La larva es de color blanco con la cabeza parda oscura, mide hasta $35 \mathrm{~mm}$ (Figura 1B). La larva construye una galería en el tronco y las ramas, donde se alimenta. Las galerías así practicadas ocasionan la marchitez y muerte de la planta. A partir del ingreso de la larva, la rama comienza a secarse y posteriormente se produce la muerte de la planta. En algunas ocasiones la mortalidad llegó al 43\% de la población, existiendo hasta seis larvas por planta. El ciclo de vida tiene una duración de hasta 70 días. 
Colaspis cf. aerea Lefevre: (Coleóptera: Chrysomelidae).

El adulto es de color verde bronce con patas color marrón amarillo (Figura 2A). Es una especie pequeña, de $6 \mathrm{~mm}$ de largo, y poco abundante; los individuos ocurren aislados o en grupos de dos a tres, en las inflorescencias y en el haz de las hojas, sin causar grandes daños. Cuando el sol es muy intenso se refugia en el envés de la hoja u otras estructuras. Fue encontrado sobre muchas solanáceas.

\section{Neoleucinodes elegantalis (Guenée) (Lepidoptera: Pyralidae).}

El adulto es una microlepidóptera de color blanco cenizo con manchas oscuras en la base y el ápice del primer par de alas, y una más clara de forma triangular en el centro; mide de 23 a $26 \mathrm{~mm}$ de envergadura alar. La larva, de color rosado claro, mide hasta $23 \mathrm{~mm}$ de largo (Figura 2B). El huevo es depositado en la superficie de los frutos verdes, luego la larva se introduce al fruto para alimentarse de pulpa y semillas. Para empupar la larva abandona el fruto dejando un orificio, que es puerta de ingreso para los patógenos. Esta mariposa fue reportada recientemente como plaga de la cocona, en la Región de Tingo María (Anteparra \& Vargas 2009).

Chromacris peruviana (Pictet \& Saussure, 1887) (Hortoptera: Acriididae)

El adulto es un saltamonte que mide hasta $33 \mathrm{~mm}$ de largo. De color verde con bandas amarillas en las patas, abdomen, tórax, cabeza y borde posterior de la antena. La ninfa de los primeros estadios es de color negro con bandas rojas, y la de los últimos tiene las mismas tonalidades que el adulto. Los individuos viven en colonias numerosas, se ha reportado hasta 105 individuos en una planta (Figura 3). Se alimentan de las hojas y, dependiendo del tamaño de la planta, en una hora pueden defoliarla totalmente. Fueron encontrados con mayor frecuencia en una plantación en la localidad de Tingo María - Región Huánuco.

\section{Manduca sexta L (Lepidoptera: Shingidae).}

El adulto es una mariposa grande y robusta, mide 87 mm de expansión alar, con el ala anterior de color cenizo oscuro, larga y estrecha, de forma triangular. El ala posterior es más clara y pequeña, con tres fajas negras, y de forma cónica. El abdomen tiene manchas dorsales amarillas, dos por cada segmento. La larva mide $100 \mathrm{~mm}$ de largo, con la piel lisa, de color verde con bandas oblicuas de color blanco y un cuerno en el último segmento abdominal. La larva vive en forma aislada, alimentándose de la hoja hasta completar su desarrollo, para empupar desciende hasta el suelo. La defoliación provocada por esta especie puede ser muy importante para una población cultivada. Fue encontrada tanto en Tingo María como en Loreto.

\section{Edessa rufomarginata De Geer (Hemíptera: Pentatomidae).}

Es una chinche grande, mide de 17 a $20 \mathrm{~cm}$ de largo, con cuerpo de forma oval, cabeza más ancha que larga, la parte dorsal de cuerpo de color verde oscuro, y la parte ventral, incluidas las patas y la antena, de color rojo (Figura 4). Conocida como plaga de las Solanáceas, es ocasionalmente encontrad en el camote, el arroz, el frijol y el maíz. Los huevos son dejados sobre las hojas, los estadios inmaduros y maduros se alimentan de la savia de la fruta.

Arvelius porrectispinus Breddín (Hemíptera: Pentatomidae).

El adulto mide de 13 a $16 \mathrm{~mm}$ de largo, son de color verde pálido, el scutellum y pronotum muestra pequeñas espinas de color negruzco. Son poco visibles en las hojas. Las ninfas viven en grupos de hasta 30 individuos, pero el adulto es solitario.

Corythaica cyathicollis Costa (Hemíptera: Tingidae).

El adulto y la ninfa son cenizos, poco visibles, se encuentran en grupos en el envés de las hojas. Los adultos miden de 1.5 a $3 \mathrm{~mm}$ de longitud. El síntoma de ataque de esta especie consiste en manchas amarillentas en las hojas. La mancha se torna progresivamente marrón y aumenta de acuerdo al número de insectos. La hoja se seca, se enrolla y cae en pedazos. En ciertos casos las colonias pueden contener más de 100 individuos. El ciclo de vida es de aproximadamente de 28 a 35 días.

\section{Dismicoccus brevipes (Homóptera: Pseudoccidae).}

Conocida como queresa de la piña. La hembra adulta mede $3 \mathrm{~mm}$ de largo, está cubierta por secreciones blancas parecidas a algodón, con apéndices del mismo color alrededor del cuerpo. El adulto no se mueve, puede estar agrupado en densas colonias, en las raíces, plato radicular y parte basal del tallo. Produce necrosis en las partes afectadas, provocando la muerte masiva de las plantas.

La humedad es un factor que favorece el establecimiento y desarrollo de la plaga. Esta queresa está asociada con hormigas del género Solenopsis sp., la plaga se detecta por la presencia de hormigas en la planta. 
Planococcus pacificus Cox (Homóptera: Pseudococcidae)

Se encuentra en colonias en los frutos medios maduros y maduros, cálices, pedúnculos y brotes. Las colonias están recubiertas por capas de tierra construidas por hormigas del género Solenopsis sp. Dada la baja presencia en la planta esta especie no significa mayores problemas para el cultivo.

\section{Aphis gossypii Glover (Homóptera: Aphididae).}

Conocido como pulgón del algodonero. Es un insecto pequeño, áptero u alado, mide hasta $1.8 \mathrm{~mm}$ de longitud, de color de amarillo claro a verde oscuro. Vive en densas colonias chupando la savia de los brotes, frutos pequeños e inflorescencias. Produce marchitamiento de las flores y retrasa el crecimiento de los frutos. El insecto secreta sustancias azucaradas que favorecen el establecimiento del hongo fumagina, lo que impide su desarrollo. Dependiendo de la densidad, la producción puede ser afectada en gran medida.

\section{Atta cephalotes (Hymenoptera: Formicidae) \\ Atta sexdens (Hymenóptera: Formicidae)}

El adulto (obrero, soldados etc.) es una hormiga de color castaño a castaño oscuro, caracterizada por tres pares de espinas en la parte dorsal del tórax. Su tamaño es muy variable, de $6 \mathrm{~mm}$ a $14 \mathrm{~mm}$. En $A$. sexdens, cuando la cabeza de un soldado es cogida entre los dedos, libera un fuerte olor a limón y en Atta cephalotes libera un olor repugnante. Las especies del género Atta viven en colonias en nidos subterráneos, que pueden llagar a tener de 7 a $8 \mathrm{~m}$ de profundidad y cubrir una área de 50 a $100 \mathrm{~m}^{2}$. Están constituidos por decenas o, cuando son antiguos, por centenas de cámaras subterráneas, ligadas entre sí por numerosos túneles de comunicación y huecos de salida. Las hormigas cortan pedazos de hojas, que transportan hasta su nido siguiendo caminos bien visibles. El daño es muy caracterizado. Una cama de producción de cocona puede ser destruida totalmente en una noche

\section{Melodogyne sp. (Nematodo)}

Es un nematodo que forma agallas características en las raíces y cuello del tallo de la planta (Figura 5). Algunas veces se observaron las agallas en la parte aérea. Estas agallas interfieren con la absorción del agua y el transporte de nutrientes por la planta, ocasionando retraso en el desarrollo, marchites de la planta, baja producción y amarillamiento de los frutos. La hembra ponen los huevos en el suelo, una vez emergidos el nematodo entra a la planta por la raíces y estimula la formación agallas. En todos los lugares donde se registró el nematodo, los suelos fueron arenosos y húmedos.
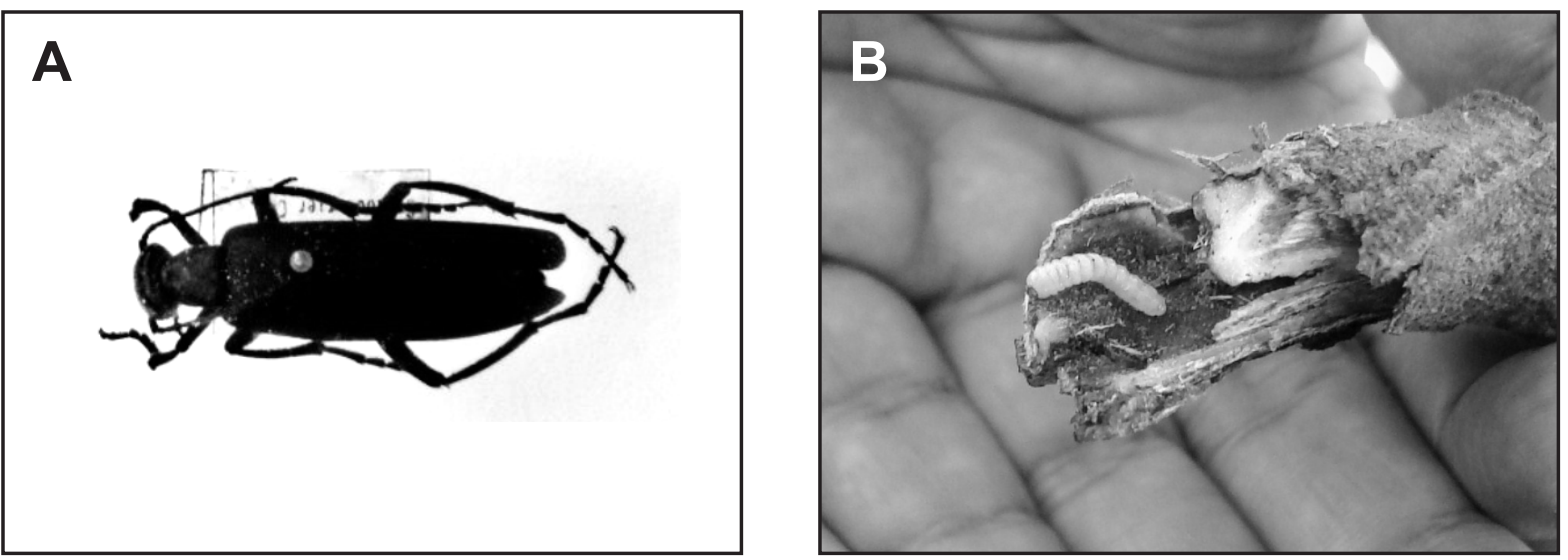

Figura 1. A) Adulto de Epicauta pestifera (Coleoptera: Meloidae), B) Larva de Alcidion deletum (Coleóptera: Cerambicidae) provocando galerias en ramas de Solanum sessiliforum. 

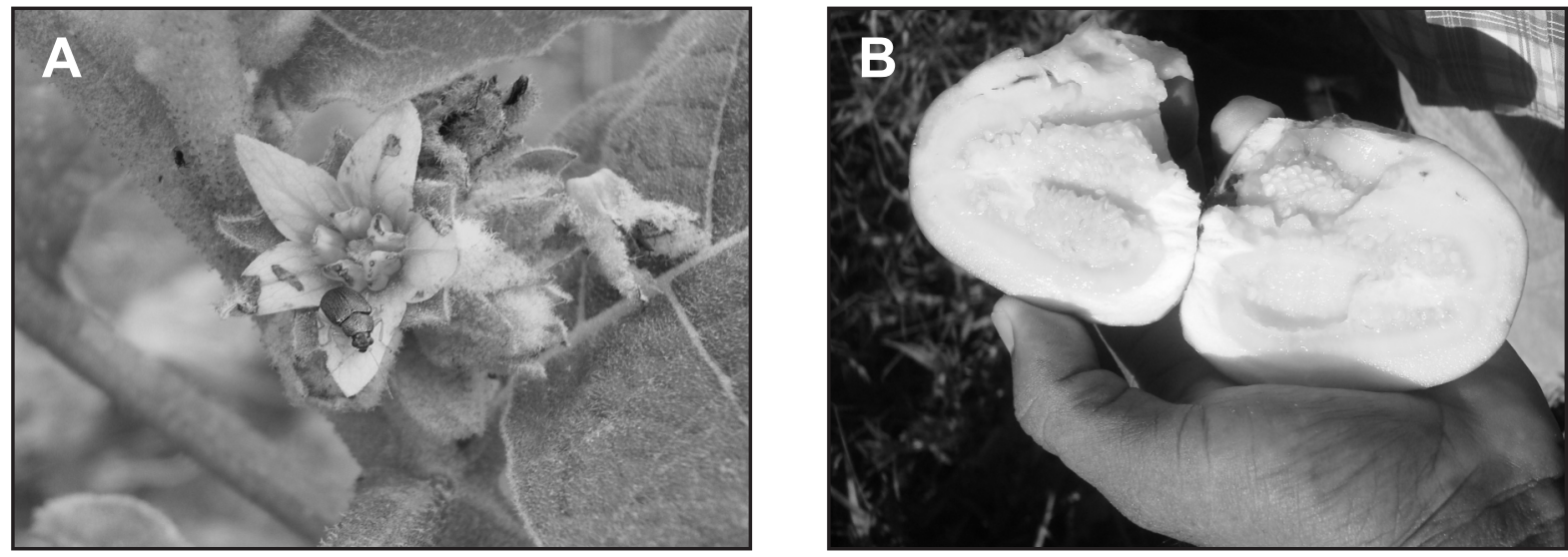

Figura 2. A) Adulto de Colaspis cf. aerea (Coleóptera: Chrysomelidae) sobre la inflorescencia de Solanum sessiliforum; B) Daños provocados por la larva de Neoleucinodes elegantalis (Lepidoptera: Pyralidae) sobre los frutos de Solanum sessiliforum.

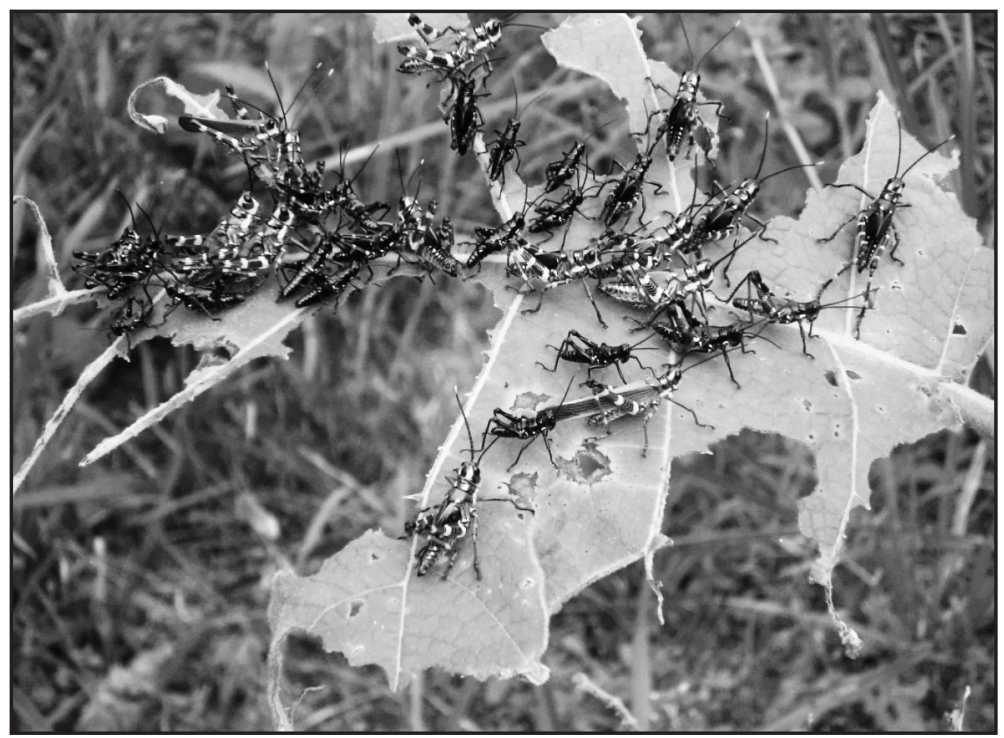

Figura 3. Colonias de Chromacris peruviana (Hortoptera: Acriididae) provocando daños en las hojas de Solanum sessiliforum. 
FOLIA

Amazónica

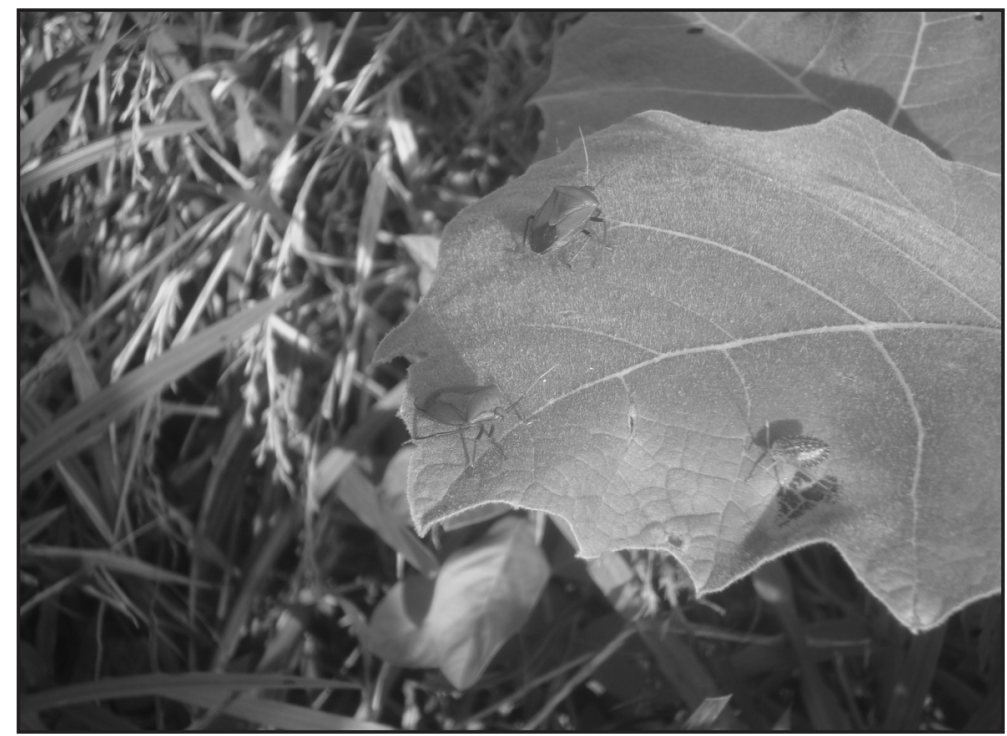

Figura 4. Adulto de Edessa rufomarginata (Hemíptera: Pentatomidae) sobre hojas de Solanum sessiliforum.

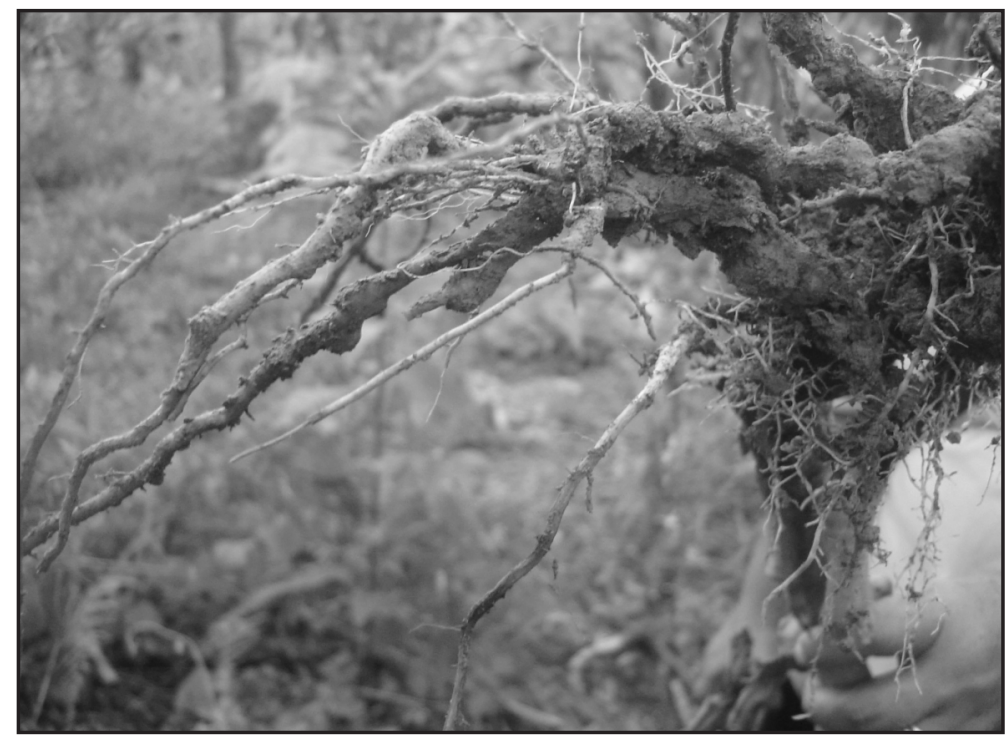

Figura 5. Daños en las raíces de Solanum sessiliforum, provocado por el nematodo del género Melodogyne 


\section{CONCLUSIÓN}

La cocona es un importante recurso genético de la Amazonía, con buenas potencialidades para la explotación industrial y comercial. Sin embargo, el desarrollo del cultivo está limitado por la presencia de numerosos invertebrados fitófagos, que limitan significativamente la producción de frutos y muchas veces matan a la planta. Durante el estudio fueron registradas cinco especies fitófagos de importancia económica, las mismas que se constituyen en nuevas plagas para la cocona en la región Amazónica. El trabajo es el primer registro de insectos fitófagos asociados a la cocona en la Amazonía peruana, es de gran importancia para profundizar los estudios, sobre la biología y ecología de los insectos, que servirán de base para el desarrollo de un programa de Manejo Integrado de Plagas del cultivo en la Región.

\section{AGRADECIMIENTO}

Expresamos nuestro agradecimiento los señores agricultores de las comunidades donde se desarrolló el trabajo, por las facilidades para visitar sus parcelas. Al Técnico Wilson Gonzáles por su apoyo en el trabajo de campo y cuidado de los ejemplares durante la cría de los inmaduros en el laboratorio.

\section{BIBLIOGRAFIA CITADA}

Anteparra, M.E.; Vargas, K. 2009. Primer registro para el Perú del perforador del fruto de cocona Neoleucinodes elegantalis (Geneé) (Lepidoptera: Pyralidae). L Convención Nacional de Entomología. Tacna- Perú. 88 pp.
Couturier, G. 1988. Alguns insetos do cubiu (Solanum sessiliforum Dunal var sessiliforum Dunal, Solanaceae) na região de Manaus - AM. Acta amazônica, 18 (3-4): 93-103.

Shultes, R.E. 1984. Amazonian cultigens and their northward migrations in pre-Colombia times. In: Pre-historic plant migration. Cambridge; Harvard University Press, pag. 19-38.

Yuyama, L.K.O.; Aguiar, J.P.; Macedo, S.H.M.; Gioia, T.; Silva Filho, D.F.1997. Composición centesimal de diversas poblaciones de cocona (Solanum sessiliflorum Dunal) de la Estación Experimental del Instituto Nacional de Investigaciones del Amazonas, INPA. In: Anuales del II Simposio Latino Americano de Ciencias de Alimentos. Campinas, S. P., Brasil.

Salick, J. 1987. Cocona (Solanum sessiliflorum) production and breeding potentials of the peachtomato. In: WICKENS, N.H; DAY, P. New crops for food and industry. Ed. Chapman and Hall. p. 258-264.

Silva Filho, D.F.; Anunciação Filho, C.J.; Noda, H.; Reis, O.V. 1996. Variabilidad genética en poblaciones naturales de cocona del Amazonas. Horticultura Brasileira. 14(1):9-15.

Silva Filho, D.F. 1998. Cocona (Solanum sessiliflorum Dunal): cultivo y utilización. TCA, Caracas-Venezuela. 104 pp.

Valcazar, L.E.; Carvajal, T.; Remusgo R. 2011. Mejoramiento genético de la cocona. 